\title{
QUASICONFORMAL AND BI-LIPSCHITZ HOMEOMORPHISMS, UNIFORM DOMAINS AND THE QUASIHYPERBOLIC METRIC BY
}

\author{
GAVEN J. MARTIN
}

\begin{abstract}
Let $D$ be a proper subdomain of $R^{n}$ and $k_{D}$ the quasihyperbolic metric defined by the conformal metric tensor $d \bar{s}^{2}=\operatorname{dist}(x, \partial D)^{-2} d s^{2}$. The geodesics for this and related metrics are shown, by purely geometric methods, to exist and have Lipschitz continuous first derivatives. This is sharp for $k_{D}$; we also obtain sharp estimates for the euclidean curvature of such geodesics. We then use these results to prove a general decomposition theorem for uniform domains in $R^{n}$, in terms of embeddings of bi-Lipschitz balls. We also construct a counterexample to the higher dimensional analogue of the decomposition theorem of Gehring and Osgood.
\end{abstract}

1. Introduction. We shall assume throughout that $D$ is a proper subdomain of euclidean $n$-space, $n \geqslant 2$. For such a domain we define the quasihyperbolic metric $k_{D}$ by

$$
k_{D}\left(x_{1}, x_{2}\right)=\inf _{C} \int_{C} \operatorname{dist}(x, \partial D)^{-1} d s,
$$

where the infimum is taken over all rectifiable arcs $C$ joining $x_{1}$ to $x_{2}$ in $D$. Many of the basic properties of this metric can be found in [4]. In particular geodesic curves exist for this complete metric. This does not follow from standard results in differential geometry, since even in domains with smooth boundary one can see that the defining density $\operatorname{dist}(x, \partial D)^{-1}$ need not be differentiable.

We primarily are interested in studying the geometry of the quasihyperbolic meric and its geodesics. We show that the geodesics of this metric are $C^{1,1}$, i.e. the arclength parametrisation has Lipschitz continuous derivatives. We show that this is best possible and obtain a sharp result on the euclidean curvature of such geodesics. These results are proved for a more general class of metrics, in particular metrics defined by locally Lipschitz densities. We then use these results to prove a generalisation of the decomposition theorem of Gehring and Osgood for uniform domains in $n$-space.

I wish to express my sincere thanks to F. W. Gehring for suggesting many of these problems, for many helpful ideas and simplifications throughout and for allowing me to present his proof of Theorem 3.7. I also wish to thank him and D. Herron for carefully reading the manuscript.

Received by the editors December 20, 1983 and, in revised form, October 20, 1984.

1980 Mathematics Subject Classification. Primary 30C60, 53C22.

(C)1985 American Mathematical Society $0002-9947 / 85 \$ 1.00+\$ .25$ per page 
1.1 Notation. We denote euclidean $n$-space by $R^{n}$. $B^{n}(x, r)$ will be the euclidean ball at $x$ of radius $r, \bar{B}^{n}(x, r)$ will be its closure and $S^{n-1}(x, r)$ its boundary. The euclidean distance from $x$ in $D$ to the boundary of $D$ is denoted $\operatorname{dist}(x, \partial D)$. We let $e_{1}=(1,0, \ldots, 0) \in R^{n}$.

\section{Convexity.}

2.1 Definition. We say that an arc $C$ in $D$ is c-convex with respect to balls, or simply c-convex, if for each $x$ in $D$ and $r \leqslant c \operatorname{dist}(x, \partial D), C \cap \bar{B}^{n}(x, r)$ is connected.

It is clear that each line segment and subarc of any circle of sufficiently large radius will be $c$-convex. These are the geodesics of the hyperbolic metric in a half-space and analogously we have

2.2 TheOREM. Each quasihyperbolic geodesic of $D$ is 1-convex with respect to balls in $D$.

Proof. Let $C$ be a quasihyperbolic geodesic of $D, x_{0} \in D$ and $r \leqslant \operatorname{dist}\left(x_{0}, \partial D\right)$. We suppose for contradiction that $C \cap \bar{B}^{n}\left(x_{0}, r\right)$ is not connected. We assume by translation that $x_{0}=0$. Let $C^{\prime}$ be a component of $C \backslash \bar{B}^{n}(0, r)$ such that the endpoints of $C^{\prime}$ lie on $S^{n-1}(0, r)$. Since $C^{\prime}$ is a subarc of a geodesic it also is a quasihyperbolic geodesic; we use this fact extensively throughout this paper. We get a contradiction by finding a quasihyperbolically shorter arc between the endpoints of $C^{\prime}$. Let $g: R^{n} \rightarrow R^{n}$ be the Möbius inversion in $S^{n-1}(0, r)$ and set $C^{\prime \prime}=g\left(C^{\prime}\right)$. It is clear that $C^{\prime \prime}$ has the same endpoints as $C^{\prime}$ and lies in $D$. We claim that for each $x$ in $D$

$$
\left|g^{\prime}(x)\right|<\frac{\operatorname{dist}(g(x), \partial D)}{\operatorname{dist}(x, \partial D)}
$$

so that

$$
\begin{aligned}
\int_{C^{\prime \prime}} \operatorname{dist}(x, \partial D)^{-1} d s & =\int_{C^{\prime}} \operatorname{dist}(g(x), \partial D)^{-1}\left|g^{\prime}(x)\right| d s \\
& <\int_{C^{\prime}} \operatorname{dist}(x, \partial D)^{-1} d s
\end{aligned}
$$

and $C^{\prime \prime}$ is shorter than $C^{\prime}$. Since $g$ is a Möbius inversion,

$$
\left|g^{\prime}(x)\right|=r^{2}|x|^{-2} \text {. }
$$

For $x \in C^{\prime}$ let $x^{\prime}=g(x)$ and choose $z \in \partial D$ such that $\left|z-x^{\prime}\right|=\operatorname{dist}\left(x^{\prime}, \partial D\right)$. Then $|z|^{2} \geqslant r^{2}=|x|\left|x^{\prime}\right|$ and, by the lemma below,

$$
\frac{\operatorname{dist}(x, \partial D)}{\operatorname{dist}(g(x), \partial D)} \leqslant \frac{|x-z|}{\left|x^{\prime}-z\right|} \leqslant\left(\frac{|x|}{\left|x^{\prime}\right|}\right)^{1 / 2}<\frac{|x|}{\left|x^{\prime}\right|}=\left(\frac{r}{|x|}\right)^{-2}=\left|g^{\prime}(x)\right|^{-1},
$$

as required.

2.3 Lemma. Suppose that $0, x$ and $x^{\prime}$ are collinear points in $R^{n}$ with $0<\left|x^{\prime}\right| \leqslant|x|$ and that $z \in R^{n}$ with $|z|^{2} \geqslant|x|\left|x^{\prime}\right|$. Then

$$
\frac{|x-z|}{\left|x^{\prime}-z\right|} \leqslant\left(\frac{|x|}{\left|x^{\prime}\right|}\right)^{1 / 2}
$$


Proof. Set $\left|x^{\prime}\right|=a,|x|=b$ and $|z|=c$, so $c^{2} \geqslant a b$. Let $\theta$ denote the angle between the line segments joining $x$ and $z$ to 0 . Now,

$$
\begin{aligned}
\left(\frac{|x-z|}{\left|x^{\prime}-z\right|}\right)^{2} & =\frac{b^{2}+c^{2}-2 b c \cos \theta}{a^{2}+c^{2}-2 b c \cos \theta}=\frac{b}{a}\left(\frac{b^{2}+c^{2}}{2 b c}-\cos \theta\right)\left(\frac{a^{2}+c^{2}}{2 a c}-\cos \theta\right)^{-1} \\
& =\frac{b}{a}(B-\cos \theta)(A-\cos \theta)^{-1}=\frac{b}{a}\left(1+\frac{B-A}{A-\cos \theta}\right),
\end{aligned}
$$

where $A=\frac{1}{2}(a / c+c / a)$ and $B=\frac{1}{2}(b / c+c / b)$. Since

$$
B-A=\frac{1}{2}\left(\frac{b}{c}+\frac{c}{b}-\frac{a}{c}-\frac{c}{a}\right)=\frac{1}{2}(b-a)\left(1-\frac{c^{2}}{a b}\right) c^{-1} \leqslant 0,
$$

the result follows.

We now prove that locally, quasihyperbolic geodesics are convex with respect to cones; this will later give a sharp curvature estimate. For $x \in D$ and

$$
\frac{1}{2} \operatorname{dist}(x, \partial D) \leqslant r<\operatorname{dist}(x, \partial D)
$$

we define an $r$-cone at $x, Q(x, r)$, as follows. Let $y \in D$ such that $|x-y|=$ $\operatorname{dist}(x, \partial D)$ and let $z=S^{n-1}(x, r) \cap[x, y]$. Then set $d=\operatorname{dist}(x, \partial D)$ and $Q(x, r)=$ convex span of $\left\{z, \bar{B}^{n}\left(x, \frac{1}{2}(d-r)\right) \cap S^{n-1}(z, r)\right\}$.

2.4 Proposition. Let $C$ be a quasihyperbolic geodesic of $D$. Then each subarc $C^{\prime}$ and $C$ with endpoints on $B^{n}\left(x_{0}, \frac{1}{2}(d-r)\right) \cap S^{n-1}(z, r)$ lies entirely within $Q\left(x_{0}, r\right)$.

Proof. Since $C^{\prime}$ is a geodesic, it is 1-convex and so $C^{\prime} \subset B^{n}\left(x_{0}, \frac{1}{2}(d-r)\right)$. Let $g$ be Möbius inversion in $S^{n-1}(z, r)$ and $C^{\prime \prime}=g\left(C^{\prime}\right) \subset Q\left(x_{0}, r\right) \subset D$. For $x \in C^{\prime}$ let $x^{\prime}=g(x)$ and $p \in \partial D$ such that $\left|x^{\prime}-p\right|=\operatorname{dist}\left(x^{\prime}, \partial D\right)$. Then, by the lemma below,

$$
\frac{\operatorname{dist}(x, \partial D)}{\operatorname{dist}\left(x^{\prime}, \partial D\right)} \leqslant \frac{|x-p|}{\left|x^{\prime}-p\right|}<\frac{|x-z|}{\left|x^{\prime}-z\right|}=\left|g^{\prime}(x)\right|^{-1} \text {. }
$$

This gives a contradiction, as $C^{\prime \prime}$ will be quasihyperbolically shorter than $C^{\prime}$ by (2.2).

2.5 Lemma. Let $r \leqslant d, x \in B^{n}\left(0, \frac{1}{2}(d-r)\right)$ and $z \in S^{n-1}(0, r)$. Then for any $x^{\prime} \in(x, z)$ and $p \in R^{n} \backslash B^{n}(0, d)$

$$
\frac{|x-p|}{\left|x^{\prime}-p\right|}<\frac{|x-z|}{\left|x^{\prime}-z\right|} \text {. }
$$

Proof. $B^{n}\left(\frac{1}{2}(x+z), \frac{1}{2}|x-z|\right) \subset B^{n}(0, d)$ so that for all $p \in R^{n} \backslash B^{n}(0, d)$

$$
\angle\left(x^{\prime}, p, z\right)<\angle(x, p, z) \leqslant \pi / 2 .
$$

If $\phi$ is the angle $\angle(p, z, x)$, then

$$
\frac{|x-p|}{\sin \phi}=\frac{|x-z|}{\sin \angle(x, p, z)} \text { and } \quad \frac{\left|x^{\prime}-p\right|}{\sin \phi}=\frac{\left|x^{\prime}-z\right|}{\sin \angle\left(x^{\prime}, p, z\right)} \text {. }
$$

The result now follows from (2.4).

2.6 REMARKS. We will see later that it is immaterial whether we define convexity with respect to open or closed balls. Also for convenience we have defined geodesics to be length minimizing curves. We may, more generally, define a geodesic of a 
nonsmooth conformal metric to be a locally length minimizing curve. Thus there may be more than one geodesic, in possibly more than one homotopy class, connecting two distinct points. All the results of this paper will then apply to each length minimal subarc of each geodesic. To see that not all geodesics in every homotopy class are convex with respect to balls, one need only consider the annulus in $R^{2}$ with the quasihyperbolic metric. We can see that the notion of convexity of the geodesics of the quasihyperbolic metric is an example of a more general phenomena. The condition essentially says that euclidean balls of a suitable size are geodesically convex in the metric being considered, i.e. for each pair of points in $B^{n}(x, r)$, with $r$ suitably small, any geodesic connecting this pair of points lies entirely within $B^{n}(x, r)$. We now examine this phenomena for other metric densities. The results will not be as sharp as those of Theorem 2.2 and Proposition 2.4, which we use later.

2.7 Definition. Let $q: D \rightarrow R_{+}$be a continuous function. For $x, y \in D$ let

$$
d_{q}(x, y)=\inf _{C} \int_{C} q(z) d s
$$

where the infimum is taken over all locally rectifiable arcs joining $x$ to $y$ in $D$. It is clear that $d_{q}$ defines a metric in $D$. We will call an arc for which the infimum is obtained a $d_{q}$-geodesic.

$d_{q}$-geodesics need not exist in general. However we show the following.

2.8 TheOREM. Let $q: D \rightarrow R_{+}$be a continuous density which defines a complete metric $d_{q}$. Then $d_{q}$-geodesics exist.

Proof. Let $x_{1}, x_{2} \in D$ and let $C_{j}$ be a sequence of locally rectifiable arcs for which

$$
d_{q}\left(x_{1}, x_{2}\right)=\lim _{j \rightarrow \infty} \int_{C_{j}} q(x) d s .
$$

Let $E=\left\{x \in D: d_{q}\left(x_{1}, x\right) \leqslant d_{q}\left(x_{1}, x_{2}\right)+1\right\}$. Since the metric $d_{q}$ is complete, $E$ is compactly contained in $D$. We can assume that for $j \geqslant j_{0}$

$$
\int_{C_{j}} q(x) d s \leqslant d_{q}\left(x_{1}, x_{2}\right)+\frac{1}{2} \text {. }
$$

Thus $C_{j} \subset E$ for $j \geqslant j_{0}$, since if not let $x_{3}$ be a point of $C_{j} \cap \partial E$; then

$$
d_{q}\left(x_{1}, x_{2}\right)+1=d_{q}\left(x_{1}, x_{3}\right) \leqslant \int_{C_{1}} q(x) d s \leqslant d_{q}\left(x_{1}, x_{2}\right)+\frac{1}{2},
$$

a contradiction. Now since $E$ is compact and $q$ is continuous there is an $a>0$ such that $q(x) \geqslant a$ for all $x \in E$. Thus for $j \geqslant j_{0}$,

$$
d_{q}\left(x_{1}, x_{2}\right)+1 \geqslant \int_{C_{j}} q(x) d s \geqslant a \int_{C_{j}} d s,
$$

and so the $C_{j}$ have uniformly bounded arclength. From the Helly selection principle we obtain a subsequence of the $C_{j}$ and a rectifiable arc $C$ joining $x_{1}$ to $x_{2}$ in $E \subset D$ such that

$$
d_{q}\left(x_{1}, x_{2}\right)=\lim _{k} \int_{C_{J_{k}}} q(x) d s=\int_{C} q(x) d s .
$$


Thus $C$ is a $d_{q}$-geodesic and the result follows. We note that $q$ need only have been locally bounded below.

We are interested in knowing when the geodesics of such metrics will be convex. An obvious condition would be an analogue of (2.1) for then (2.2) would imply convexity. A sufficient condition is easily seen to be:

For all $y$ and $r$ with $B^{n}(y, r) \subset D$ and $g$ a Möbius inversion in $S^{n-1}(y, r)$,

$$
\left|g^{\prime}(x)\right| \leqslant \frac{q(x)}{q(g(x))} \quad \text { for all } x \in D \backslash B^{n}(y, r) .
$$

Since $\left|g^{\prime}(x)\right|=r^{2} /|x-y|^{2}$, setting $x^{\prime}=g(x),(2.5)$ becomes

$$
r^{2} q\left(x^{\prime}\right) \leqslant|x-y|^{2} q(x) .
$$

This condition can be seen as a control on the rate of growth of $q$ along radial lines emanating from $y$. Notice that if $q$ is continuous, then the left-hand side of (2.6) is bounded while the factor $|x-y|$ on the right is increasing. This suggests that convexity is a local condition. If we can establish (2.6) for sufficiently small $r$, then the metric will be $c$-convex for sufficientiy small $c$. We will say that a metric is locally convex if for each compact set $E \subset D$ there is a positive constant $c=c(E, q)$ such that $d_{q}$ is $c$-convex on $E$, i.e. for any $x$ in $E, r \leqslant c \operatorname{dist}(x, \partial D)$ and $C$, a $d_{q}$-geodesic, $C \cap \bar{B}^{n}(x, r)$ is connected.

The following corollaries are immediate from (2.6).

2.9 COROLlary. If $d_{q}$ is a metric whose density satisfies (2.5), then $d_{q}$ is 1-convex.

2.10 Corollary. If $q$ is a Möbius invariant density, i.e. $q(x)=\left|g^{\prime}(x)\right| q(g(x))$ for $g$ as in (2.5), then $d_{q}$ is 1-convex.

2.11 Corollary. If $D$ is a bounded domain and $d$ is the metric defined by the density $\operatorname{dist}(x, \partial D)^{-p}$, then $d$ has geodesics if $p \geqslant 1$ and $d$ is 1 -convex if $1 \leqslant p \leqslant 2$.

Proof. The metric is easily seen to be complete if $p \geqslant 1$ and so Theorem 2.8 gives the existence of geodesics. These geodesics are 1-convex if $1 \leqslant p \leqslant 2$ since we did not use the inequality of Lemma 2.3 sharply in the proof of Theorem 2.2. The more general result we are seeking is the following.

2.12 Theorem. Let $q: D \rightarrow R_{+}$be a locally Lipschitz density defining a complete metric $d_{q}$. Then $d_{q}$ is locally convex.

Proof. Let $M>0$. For $x \in D$ let $r(x, M)$ denote the largest number such that

$$
B^{n}(x, r(x, M)) \subset B_{q}^{n}(x, M)=\left\{y \in D: d_{q}(x, y)<M\right\} .
$$

Since $\bar{B}_{q}^{n}(x, 2 M)$ is compactly contained in $D$ we let $c(x, M)$ and $L(x, M)$ denote respectively the largest and smallest numbers for which

(1) $q(x) \geqslant c(x, M)$ on $B_{q}^{n}(x, 2 M)$,

(2) $q(x)$ is $L(x, M)$ Lipschitz on $B_{q}^{n}(x, 2 M)$. 
On any compact set contained in $D$ it is clear that $c(x, M)$ and $r(x, M)$ are bounded below whilst $L(x, M)$ is bounded above. By Theorem 2.8, $d_{q}$-geodesics exist. Let $x \in D$ and $r<\min \{r(x, M), c(x, M) / L(x, M)\}$. Suppose that $C$ is a $d_{q}$-geodesic and that $C \cap B^{n}(x, r)$ is not connected. Let $C^{\prime}$ be a subarc of $C \backslash \bar{B}^{n}(x, r)$ with endpoints on $S^{n-1}(x, r), g$ be a Möbius inversion in $S^{n-1}(x, r)$ and $C^{\prime \prime}=g\left(C^{\prime}\right)$. Let $c=c(x, M)$ and $L=L(x, M)$. We now show that (2.6) holds along $C^{\prime}$ to get the desired contradiction from (2.2). Let $y \in C^{\prime}$ and $y^{\prime}=g(y) \in C^{\prime \prime}$. Since $C^{\prime}$ is a subarc of a geodesic it also is a $d_{q}$-geodesic. The endpoints of $C^{\prime}$ lie on $S^{n-1}(x, r) \subset B_{q}^{n}(x, M)$ and so $C^{\prime} \subset B_{q}^{n}(x, 2 M)$, since it is a shortest geodesic. We may assume that $q\left(y^{\prime}\right)>q(y)$ for otherwise (2.6) is trivial. Also

$$
y^{\prime}=r^{2} /|x-y|^{2}(y-x)+x
$$

and since $x, y$ and $y^{\prime}$ are collinear, $\left|y-y^{\prime}\right|=|y-x|-\left|y^{\prime}-x\right|$. Thus since $r L / c>1$ and $|x-y|>r$, we have $r^{2}(L / c)<|x-\mathrm{y}|$,

$$
\begin{gathered}
r^{2}(L / c)|x-y|\left(1-r^{2} /|x-y|^{2}\right)<|x-y|^{2}\left(1-r^{2} /|x-y|^{2}\right), \\
r^{2} L\left|y-y^{\prime}\right|<\left(|x-y|^{2}-r^{2}\right) q(y), \\
r^{2}\left(q\left(y^{\prime}\right)-q(y)\right)<\left(|x-y|^{2}-r^{2}\right) q(y),
\end{gathered}
$$

since $q$ is $L$-Lipschitz on $B_{q}^{n}(x, 2 M)$. This last inequality is precisely (2.6).

2.13 Corollary. If $q$ is as in 2.12 , bounded below by $c$ and is L-Lipschitz on $D$, then $d_{q}$ is locally convex with bounds given by $\min \{c / L, \operatorname{dist}(x, \partial D)\}$.

Proof. We have Theorem 2.12 for arbitrary $M$. Letting $M \rightarrow \infty$ we see that $L(x, M) \leqslant L, c(x, M) \geqslant c$ and $r(x, M) \rightarrow \operatorname{dist}(x, \partial D)$.

Our interest in these results stems from the fact, as we shall see later, that locally convex metrics have $C^{1,1}$ geodesics. In fact, we can get bounds on the euclidean curvature of the geodesics in terms of the convexity constants. In view of this, Theorem 2.12 can be seen as a sharp regularity result for such densities as Example 4.11 shows. These results also tend to indicate that the quasihyperbolic metric is the canonical metric for a domain in $R^{n}$. We complete this section by stating a trivial corollary to Theorem 2.12 which recovers some standard results in differential geometry.

2.14 Corollary. If $q$ is a $C^{p}$ density defining a complete metric and $p \geqslant 1$, then $d_{4}$ is locally convex.

\section{Decomposition.}

3.1 Definition. A domain $D \subset R^{n}$ is said to be uniform if there exist constants $a$ and $b$ such that each pair of points $x_{1}, x_{2} \in D$ can be joined by a rectifiable arc $C \subset D$ for which

$$
l(C) \leqslant a\left|x_{1}-x_{2}\right|, \quad \min _{j=1,2} l\left(C\left(x_{j}, x\right)\right) \leqslant b \operatorname{dist}(x, \partial D) .
$$

Here $l(C)$ denotes the length of $C$ and $l\left(C\left(x_{j}, x\right)\right)$ is the length of the subarc of $C$ from $x_{j}$ to $x, j=1,2$. 
We refer to [11] for the definition of a $K$-quasiconformal homeomorphism of a domain $D \subset R^{n}$ and many of the basic facts regarding quasiconformal mappings.

3.2 Definition. A $K$-quasidisk is the image of $B^{2}(0,1)$ under a $K$-quasiconformal homeomorphism of $\bar{R}^{2}$.

3.3 Remarks. For $n \geqslant 2$ Martio and Sarvas have shown that the image of $B^{n}(0,1)$ under a quasiconformal homeomorphism of $R^{n}$ is a uniform domain [8]. Recently uniform domains have been found to have many interesting applications in function theory, particularly in the theory of $B M O$ functions (e.g. [7]) and in the injectivity of local quasi-isometries (e.g. [3]). In $n=2$ there is a strong interplay between the notions of a uniform domain and that of a quasidisk. In particular, simply connected uniform domains are quasidisks and we have the following decomposition theorem of Gehring and Osgood [4, Theorem 5].

3.4 TheOREM. A domain $D \subset R^{2}$ is uniform if and only if it is quasiconformally decomposable, i.e. for each pair of points $x_{1}, x_{2} \in D$ there is a $K$-quasidisk $G \subset D$, such that $x_{1}, x_{2} \in \bar{G}$ and $K$ depends only on $a$ and $b$, the coefficients of uniformity.

This situation is very different in $R^{n}$. We show the above theorem is false if $n=3$, even for simply connected uniform domains. This is because the problems of topological flatness do not occur when $n=2$.

3.5 Definition. A homeomorphism $f: D \rightarrow D^{\prime}$ is said to be $L$-bi-Lipschitz if for every $x, y \in D$ we have

$$
\frac{1}{L}|x-y| \leqslant|f(x)-f(y)| \leqslant L|x-y| .
$$

3.6 Remarks. It is not difficult to show that if $D$ is a uniform domain and $f: D \rightarrow D^{\prime}$ is $L$-bi-Lipschitz, then $D^{\prime}$ is a uniform domain. In fact if $D$ is $a, b$ uniform, then $D^{\prime}$ is $a L, b L^{2}$ uniform. Also it is easy to see that $B^{n}(0,1)$ is a uniform domain. Gehring and Osgood have shown that a domain is uniform if and only if (3.1) holds for each quasihyperbolic geodesic [4, Corollary 2]. We shall use this fact extensively later.

We base our counterexample to the 3-dimensional analogue of Theorem 3.4 on a bi-Lipschitz version of the classical Fox-Artin ball. The existence of this bi-Lipschitz ball was first recognized by Gehring; though it has often been cited there is no proof in print. The outline given here is based on his ideas.

3.7 THEOREM. There is a bi-Lipschitz homeomorphism $f: \bar{B}^{3} \rightarrow R^{3}$ such that $f\left(\bar{B}^{3}\right)$ is not topologically flat. In particular $f$ cannot be extended to a homeomorphism of $R^{3}$.

OUTLINE OF PROOF. Let $C$ be the smooth arc in $R^{3}$ illustrated below. Let $t>0$ and let $N(t)$ be a smooth regular neighborhood of $C$ of radius $t$. Then it is clear by compactness and smoothness that, after possibly restricting to a smaller $t$, there is an $L_{1}$-bi-Lipschitz homeomorphism $g:[-1,0] \times B^{2}(t) \rightarrow N(t)$; we may further assume that $g$ is the identity on $\{[-1,-7 / 8] \cup[-1 / 8,0]\} \times B^{2}(t)$. One must imagine that $N(t)$ sits in three space in such a way that if $N^{\prime}=\left\{\frac{1}{2}(x+1): x \in N(t)\right\}$, then $N$ and $N^{\prime}$ link in $R^{3}$ and intersect only at the end $\{0\} \times B^{2}(t)$. We iterate this 
construction to obtain an infinitely linked ball. Let

$$
\begin{gathered}
G=\left\{\left(s, \frac{1}{2} s x\right):(s, x) \in[-1,0] \times B^{2}(t)\right\}, \\
G_{n}=\left\{(s, x) \in G:-2^{-n} \leqslant s \leqslant-2^{-n-1}\right\} .
\end{gathered}
$$

Identifying $R^{3}$ with $R \times R^{2}$ we define $h_{n}: R^{3} \rightarrow R^{3}$ by

$$
h_{n}((s, x))=2^{n+1}(s, x)+e_{1} .
$$

Then $h_{n}\left(G_{n}\right) \subset[-1,0] \times B^{2}(t)$ and

$$
h_{n}\left(-2^{-n-j} \times B^{2}\left(2^{-n-1-j} t\right)\right)=\{j-1\} \times B^{2}(t), \quad j=0,1 .
$$

This later condition implies that the ends of $G_{n}$ are mapped to the ends of $[-1,0] \times B^{2}(t)$.

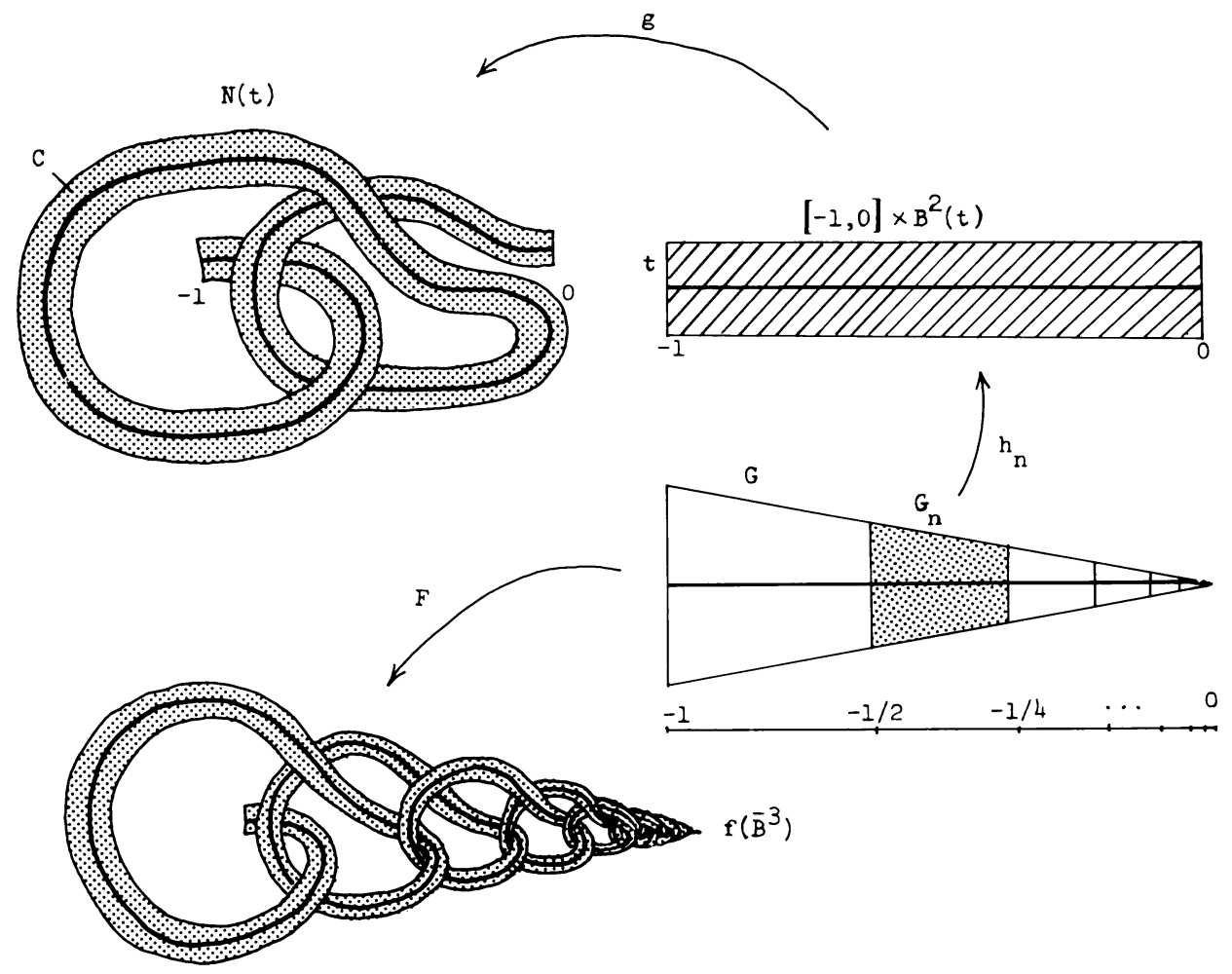

Now define $F: G \rightarrow R^{3}$ by $\left.F\right|_{G_{n}}=\left.h_{n}^{-1} g h_{n}\right|_{G_{n}}$. Since $g$ is the identity on $\{-1,0\} \times$ $B^{2}(t)$ we see that $F$ is the identity on a neighbourhood of $\left\{-2^{-n}\right\} \times B^{2}\left(2^{-n-1} t\right)$ for each $n$. These sets are the 'ends' of the $G_{n}$ and so $F$ is easily seen to be a well-defined homeomorphism. We now outline why $F$ is bi-Lipschitz. Similar techniques are used in our proof of the decomposition theorem in $R^{n}$, Theorem 5.1, and so we do not go into too much detail. Since $g$ is $L_{1}$-bi-Lipschitz, $F$ is $L_{1}$-bi-Lipschitz on each $G_{n}$ for the maps $h_{n}$ are similarities and conjugation by similarities does not alter the Lipschitz constants. Since $F$ is the identity on a neighbourhood of $G_{n} \cap G_{n-1}$ it is 
easy to see that there is a constant $L_{2}$ such that $F$ is $L_{2}$-bi-Lipschitz on each $G_{n} \cup G_{n-1}$ for all $n$. Now the convexity of $G$ yields the right inequality of (3.2) and estimates using the triangle inequality yield the left inequality of (3.2). One can now see that there is an $L_{3}$-bi-Lipschitz homeomorphism $h: B^{3} \rightarrow G$, we claim that the desired map $f$ is $F h: B^{3} \rightarrow R^{3}$. To see that $f\left(B^{3}\right)$ is not topologically flat we observe that $f\left(B^{3}\right)$ is essentially the same as Example 2.4.6 in [10]. Alternatively one can see that $f\left(B^{3}\right)$ is not one-locally coconnected at $F(0)$, i.e. $f\left(B^{3}\right)$ is not locally homotopically trivial at $F(0)$, and this is a necessary condition for flatness [2].

3.8 EXAMPLE. Theorem 3.4 does not hold in $R^{3}$.

Proof. Let $f$ be the bi-Lipschitz map of Theorem 3.7. Then by Remark 3.6 $f\left(B^{3}(0,1)\right)$ is uniform; we can assume by rotation that $f\left(e_{1}\right)$ is the wild point of $f\left(\bar{B}^{3}\right)$. Suppose for contradiction that 3.4 holds in $R^{3}$. Then there would be a constant $K$ and, corresponding to the points 0 and $(1-1 / n) e_{1}$, a sequence of $K$-quasiconformal homeomorphisms $\left\{g_{n}\right\}$ of $R^{3}$ such that $g_{n}(\infty)=\infty$ and $f(0)$, $f\left((1-1 / n) e_{1}\right) \in g_{n}\left(B^{3}(0,1)\right) \subset f\left(B^{3}(0,1)\right)$. By the compactness of such families of $K$-quasiconformal homeomorphisms we can find a subsequence of the $\left\{g_{n}\right\}$ which converges, uniformly in the spherical metric, to a $K$-quasiconformal homeomorphism $g: R^{3} \rightarrow R^{3}$. Since $g_{n}\left(B^{3}\right) \subset f\left(B^{3}\right)$ for all $n$, we must have $g\left(B^{3}\right) \subset f\left(B^{3}\right)$ and since $f\left(e_{1}\right) \in g\left(S^{n-1}\right)$ we can assume by a rotation that $f\left(e_{1}\right)=g\left(e_{1}\right)$. This together with the above implies that $g\left(B^{3}\right)$ has the same wild behaviour as $f\left(B^{3}\right)$ at $e_{1}$. This is a contradiction for $g$ is a homeomorphism of $\bar{R}^{3}$ and hence tame.

We discuss the case $n \geqslant 4$ in $\S 5$. Next we prove a version of Theorem 3.4 for arbitrary domains in $R^{n}$. By our example there can be no constant $K$ independent of the points, so we find a bound on $K$ in terms of their quasihyperbolic distance.

3.9 Lemma. Let $x \in D, r=\frac{1}{2} \operatorname{dist}(x, \partial D)$ and $0<\varepsilon<1$, so $B=B^{n}(x, r) \subset D$. Then for all $y$ satisfying $|x-y| \leqslant \varepsilon r$,

$$
k_{B}(x, y) \leqslant 2(1-\varepsilon)^{-2} k_{D}(x, y) .
$$

Proof. Let $C$ be a quasihyperbolic geodesic from $x$ to $y$. By convexity with respect to balls, $C \subset B^{n}(x, r \varepsilon)$. Now

$$
k_{B}(x, y) \leqslant \int_{C} \operatorname{dist}(z, \partial D)^{-1} d s,
$$

for $k_{B}$ is found as the infimum over all such arcs. Next for $z \in C$,

$$
\begin{aligned}
\operatorname{dist}\left(z, S^{n-1}(x, r)\right) & =r-|z-x| \geqslant r(1-\varepsilon)=\frac{1}{2}(1-\varepsilon) \operatorname{dist}(x, \partial D) \\
& \geqslant \frac{1}{2}(1-\varepsilon) \frac{\operatorname{dist}(z, \partial D)}{(1+\varepsilon / 2)} \geqslant \frac{1}{2}(1-\varepsilon)^{2} \operatorname{dist}(z, \partial D) .
\end{aligned}
$$

Putting this in the above integral and recognizing that $C$ is a quasihyperbolic geodesic yields the desired result.

3.10 Lemma. Let $x, y, z \in D$ with $k_{D}(x, y) \leqslant a \leqslant \frac{1}{4}$ and $\log 2<k_{D}(x, z)$. Then there is a quasiconformal homeomorphism $f: R^{n} \rightarrow R^{n}$ with the following properties:

(1) $f(x)=y, f(z)=z$.

(2) $f: R^{n} \backslash D \rightarrow R^{n} \backslash D$ is the identity.

(3) $\log \left(K_{I}(f)\right) \leqslant 4\left(3-2 e^{a}\right)^{-2} k_{D}(x, y)$. 
Proof. Let $d=\operatorname{dist}(x, \partial D), r=\frac{1}{2} d$ and $B=B^{n}(x, r)$. Then by Lemma 3.1 of [5] there is a quasiconformal homeomorphism $f: \bar{R}^{n} \rightarrow \bar{R}^{n}$ such that $f(x)=y$, $f: \bar{R}^{n} \backslash B \rightarrow \bar{R}^{n} \backslash B$ is the identity and $\log \left(K_{I}(f)\right) \leqslant h_{B}(x, y)$. Here $K_{I}(f)$ is the inner dilation of $f$ and $h_{B}$ is the hyperbolic metric of constant negative curvature -1 in $B$. By comparing densities one easily sees that $h_{B}(x, y) \leqslant 2 k_{B}(x, y)$ and by [4, Lemma 2.1],

$$
\log (1+|x-y| / d) \leqslant k_{D}(x, y) \leqslant a
$$

implies

$$
|x-y| \leqslant 2\left(e^{a}-1\right) r=\varepsilon r, \quad 0<\varepsilon<1 .
$$

Thus by Lemma 3.9 we have

$$
\log \left(K_{I}(f)\right) \leqslant 4\left(1-2\left(e^{a}-1\right)\right)^{-2} k_{D}(x, y) .
$$

To see $f(z)=z$ we need only show $z \notin B$. Suppose that $z \in B$. Then the line segment joining $x$ to $z$ lies in $D$ and

$$
\begin{aligned}
k_{D}(x, z) & \leqslant \int_{0^{\circ}}^{|x-z|}(\operatorname{dist}(x, \partial D)-t)^{-1} d t=\log d-\log (d-|x-z|) \\
& <\log (d /(d-r))=\log 2,
\end{aligned}
$$

a contradiction.

3.11 TheOREM. Let $x, y \in D$. Then there is a quasiconformal homeomorphism $F: R^{n} \rightarrow R^{n}$ such that $x, y \in F\left(\bar{B}^{n}\right) \subset D$ and $\log \left(K_{I}(F)\right) \leqslant 4 k_{D}(x, y)$.

Proof. We iterate Lemma 3.10. Let $d=\operatorname{dist}(x, \partial D)$. Now

$$
\log (1+|x-y| / d) \leqslant k_{D}(x, y) \leqslant \log 2
$$

implies that $|x-y| \leqslant d$ and $x, y$ both lie in $\bar{B}^{n}(x, d)$ and so we can assume that $k_{D}(x, y)>\log 2$. Let $C$ be a quasihyperbolic geodesic from $x$ to $y$ and fix $0<a<\frac{1}{4}$. Let $y=y_{0}, y_{1}, \ldots, y_{m}$ be a sequence of points on $C$ such that

$$
k_{D}\left(y_{j-1}, y_{j}\right)=a, \quad j=1,2, \ldots, m,
$$

and that

$$
k_{D}\left(x, y_{m}\right) \leqslant \log 2<k_{D}\left(x, y_{m-j}\right), \quad j=1, \ldots, m-1 .
$$

For $j<m$ Lemma 3.10 yields a quasiconformal homeomorphism $g_{j}$ such that $g_{j}(x)=x, g_{j}\left(y_{j-1}\right)=y_{j}$ and

$$
\log \left(K_{I}\left(g_{j}\right)\right) \leqslant 4\left(3-2 e^{a}\right)^{-2} k_{D}\left(y_{j}, y_{j-1}\right) .
$$

Next set $G_{a}=g_{m} \cdot g_{m-1} \cdots g_{1}$. Then $G_{a}(y)=y_{m} \in B^{n}(x, d)$. It is clear that $G_{a}$ is a homeomorphism of $R^{n}$ and that $G_{a}$ is the identity on $R^{n} \backslash D$. Also

$$
\log \left(K_{I}\left(G_{a}\right)\right) \leqslant 4\left(3-2 e^{a}\right)^{-2} \sum_{j=1}^{m} k_{D}\left(y_{j}, y_{j-1}\right) \leqslant 4\left(3-2 e^{a}\right)^{-2} k_{D}(x, y) .
$$

Again by the compactness of such families of quasiconformal mappings, there is a uniformly convergent subsequence of the $\left\{G_{a}\right\}$, as $a \rightarrow 0$, converging to a quasiconformal homeomorphism $G: R^{n} \rightarrow R^{n}$ and $\log \left(K_{I}(G)\right) \leqslant 4 k_{D}(x, y)$. If we let $g$ be 
the similarity mapping of $R^{n}$ mapping $B^{n}(0,1)$ onto $B^{n}(x, d)$, then it is clear that $F=G^{-1} \cdot g: R^{n} \rightarrow R^{n}$ is the desired quasiconformal map.

3.12 Remarks. The bound on $K_{I}(F)$ in Theorem 3.11 is probably not sharp. To obtain a lower bound when $n=3$, consider the domain

$$
D=R^{3} \backslash\left\{(x, y, z): x^{2}+y^{2} \geqslant 1, z=0\right\} .
$$

Letting $z_{1}=(0,0, r)$ and $z_{2}=(0,0,-r)$ one can show, as in [6, Theorem 6.2], that if $G$ is a $K$-quasidisk lying in $D$ and containing $z_{1}$ and $z_{2}$, then $K \geqslant \pi^{-1} \log r$. The quasihyperbolic geodesic between $z_{1}$ and $z_{2}$ is the line segment $\{(x, y, z): x=0$, $|y| \leqslant r\}$, whence

$$
k_{D}\left(z_{1}, z_{2}\right)=2 \log \left(r+\left(r^{2}+1\right)^{1 / 2}\right) .
$$

Thus

$$
\frac{K_{I}(F)}{k_{D}\left(z_{1}, z_{2}\right)} \rightarrow \frac{1}{2 \pi} \quad \text { as } r \rightarrow \infty .
$$

This is probably the sharp order for $K_{I}(F)$ and this result is easily generalized to $R^{n}$. We note that in a uniform domain [4, Theorem 1],

$$
k_{D}(x, y) \leqslant c \log (1+|x-y| / \operatorname{dist}(x, \partial D))(1+|x-y| / \operatorname{dist}(y, \partial D)),
$$

where $c$ is a constant depending only on $a, b$ and $n$. Thus, in a uniform domain, we have the bound

$$
K_{I}(F) \leqslant(1+|x-y| / \operatorname{dist}(x, \partial D))^{c}(1+|x-y| / \operatorname{dist}(y, \partial D))^{c} .
$$

The main feature of this bound is that is depends only on the distance between the points, their respective distances to the boundary of $D$, and the constants of uniformity.

\section{The differentiability of convex curves.}

4.1 Definition. A set of $n+1$ vectors $x_{0}, x_{1}, \ldots, x_{n}$ is said to be in general position if any $n$ of them span $R^{n}$.

We denote by $\left(x_{i_{1}}, x_{i_{2}}, \ldots, x_{i_{p}}\right)$ the convex span of $x_{i_{1}}, x_{i_{2}}, \ldots, x_{i_{p}}$. If $x_{0}, x_{1}, \ldots, x_{n}$ are in general position, then $\left(x_{i_{1}}, \ldots, x_{i_{p}}\right)$ is a nondegenerate $p-1$ simplex and so $x_{i_{1}}, \ldots, x_{i_{p}}$ are in general position.

4.2 LemMA. Let $x_{0}, x_{1}, \ldots, x_{n}$ be in general position and suppose that $\left|x_{i}\right|=r$ for all $i=1, \ldots, n$. Then for each $x \in\left(x_{0}, x_{1}, \ldots, x_{n}\right)$,

$$
\min _{i}\left|x_{i}-x\right| \leqslant r
$$

and we have equality if and only if $x=0$.

Proof. If $x=0$ the result and equality are clear, so we assume $x \neq 0$ and use induction on $n$. The result is trivial if $n=1$. Let $A=\left(x_{0}, x_{1}, \ldots, x_{n}\right)$ be a nondegenerate $n$ simplex, and $A^{i}=\left(x_{0}, \ldots, x_{i-1}, x_{i+1}, \ldots, x_{n}\right)$ the $i$ th face of $A$. Since $A$ is compact and $x \neq 0$, there is an $a \geqslant 1$ so that

$$
a x \in \partial A=\bigcup_{i} A^{i}
$$


Assume that $a x \in A^{k}$. Since $x_{1}, \ldots, x_{k-1}, x_{k+1}, \ldots, x_{n}$ span $R^{n}$ they lie in a codimension one hyperplane $T$, not containing the origin. Now, for $i \neq k, x_{i}$ lies in $T \cap S^{n-1}(0, r)=S$ and it is easy to see that $S$ is a codimension two sphere of radius $r_{0}<r$. Identifying $T$ with $R^{n-1}$ and $S$ with $S^{n-2}\left(0, r_{0}\right)$ and using the induction hypothesis we have $\min _{i \neq k}\left|a x-x_{i}\right| \leqslant r_{0}$. Hence there is a $j$ with $\left|x_{j}-a x\right| \leqslant r_{0}$. Thus $\bar{B}^{n}\left(x_{j}, r\right)$ contains 0 and $a x$ as interior points and hence the line segment $[0, a x]$. Since $a \geqslant 1, x$ lies on this segment and the proof is complete.

Note. Henceforth we assume, for convenience, that $c \leqslant 1$.

4.3 TheOREM. Let $C \subset D$ be a locally rectifiable arc which is $c$-convex with respect to balls in $D$ and parametrized by arclength. Then

(1) C has Lipschitz continuous derivatives.

(2) If $x, y \in C,|x-y|<(c / 2) \operatorname{dist}(x, \partial D)$ and if $\phi$ is the angle between the normal hyperplanes to $C$ at $x$ and $y$, then

$$
\sin (\phi) \leqslant 4|x-y| \operatorname{dist}(x, \partial D)^{-1} .
$$

Proof. We first show that $c$-convexity implies that there is a well-defined normal hyperplane to $C$ at $x$. The Lipschitz condition follows from (2), for if we parametrize $C$ by arclength, then $2 \sin \left(\frac{1}{2} \phi\right)=\left|C^{\prime}(t)-C^{\prime}(s)\right|$, where $C(t)=x$ and $C(s)=y$, and for $\phi$ near zero, $2 \sin \left(\frac{1}{2} \phi\right)$ and $\sin (\phi)$ are comparable; the details appear later. Let $x_{0} \in C$, not an endpoint. Then by translation and reparametrisation, if necessary, we can assume that $x_{0}=0$ and that $C:[-1,1] \rightarrow D$ with $C(0)=x_{0}=0$. Let $d=\operatorname{dist}\left(x_{0}, \partial D\right)$ and $r=\frac{1}{2} c d$. For any $x \in S^{n-1}(0, r), B^{n}(x, r)$ contains the origin as a boundary point and we have the following trichotomy:

(a) $B^{n}(x, r) \cap C=\varnothing$,

(b) there is a $t>0$ such that $|C(t)-x| \leqslant r$,

(c) there is a $t<0$ such that $|C(t)-x| \leqslant r$.

Then $0 \notin B^{n}(x, r) \subset D$ and $r \leqslant c \operatorname{dist}(x, \partial D)$ so that $B^{n}(x, r)$ cannot contain points $C\left(t_{1}\right)$ and $C\left(t_{2}\right)$ with $t_{1}<0<t_{2}$ by the $c$-convexity of $C$. Also $\operatorname{dist}\left(z, S^{n-1}(r)\right)<r$ if $z \in B^{n}(r) \backslash\{0\}$, so the above situation is exhaustive. Let $E_{0}, E_{+}$and $E_{-}$be the subsets of $S^{n-1}(r)$ satisfying (a), (b) or (c) respectively. We have shown

$$
E_{0} \cup E_{+} \cup E_{-}=S^{n-1}(r) \quad \text { and } \quad E_{0} \cap E_{+}=E_{0} \cap E_{-}=E_{+} \cap E_{-}=\varnothing .
$$

It is clear that $E_{+}$and $E_{-}$are nonempty open subsets of $S^{n-1}(r)$ and so $E_{0}$ is a closed nonempty separating set in $S^{n-1}(r)$.

4.4 Proposition. The set $E_{0}$ described above is a codimension two sphere of radius $r$ and thus is centred at $x_{0}=0$.

Proof. Let $m$ be the rank of a maximal set of independent vectors in $E_{0}$. Suppose first that $m \leqslant n-2$. Then $E_{0}$ lies in a hyperplane $T$ of codimension two and so $E_{0} \subset T \cap S^{n-1}(r)$ has codimension two in $S^{n-1}(r)$ and hence cannot separate. Next suppose that $m=n$. Let $y_{1}, \ldots, y_{n}$ be such a maximal set of vectors. Set

$$
y_{0}=\frac{-r\left(y_{1}+y_{2}+\cdots+y_{n}\right)}{\left|y_{1}+y_{2}+\cdots+y_{n}\right|} \text {. }
$$


Then it is easy to see that $y_{0}, y_{1}, \ldots, y_{n}$ are in general position. Let $A=\left(y_{0}, y_{1}, \ldots, y_{n}\right)$. Then $v=n^{-1}\left(y_{1}+\cdots+y_{n}\right)$ is the barycentre of $A^{0}$ and so interior to this face. Since $y_{0}=-r v /|v|$, the line segment $\left(v, y_{0}\right)$ lies inside $A$ and so the origin, which lies on this segment, is an interior point of $A$. By Lemma 4.2, for any $x \in A, x \neq 0,(4.1)$ holds with strict inequality, so we have $\left|x-y_{j}\right|<r$ for some $j$. Thus $A \subset$ $\bigcup_{j=0}^{n} \bar{B}^{n}\left(y_{j}, r\right)$. But $y_{j} \in E_{0}$ for $j=1,2, \ldots, n$ and $y_{0}$ lies in only one of $E_{0}, E_{+}$or $E_{-}$. Thus $A$ can contain points $C(t)$ with $t \leqslant 0$ or $0 \leqslant t$ but not both. This is a contradiction since $A$ is a neighbourhood of $x_{0}$, which is not an endpoint of $C$. Finally we conclude that $m=n-1$, so that $E_{0}$ lies in a codimension one hyperplane $T$ containing the origin and since $E_{0}$ separates, $E_{0}=T \cap S^{n-1}(r)$.

Proof of Theorem 4.3 continued. Proposition 4.4 enables us to define a unique "normal" hyperplane to $C$ at $x_{0}$ as the unique codimension one hyperplane through $E_{0}$. For $x \in C$ we denote this choice by $T_{x}$. To see that $T_{0}$ is indeed the normal hyperplane to $C$, we need only show that near $x_{0}$ we can parametrise $C$ so that $(d / d t) C \neq 0$. We require the following geometric results to obtain this parametrisation.

4.5 Lemma. If $C$ is c-convex with respect to balls in $D$ and if $r<c \operatorname{dist}(x, \partial D)$, then $C \cap S^{n-1}(x, r)$ contains at most two points.

Proof. Suppose not. We can assume by translation that $x=0$. Let $u, v$ and $w$ be an ordered triple of points of $C$ with $|u|=|v|=|w|=r$, thus $(u, v, w)$ is a nondegenerate triangle. Let $0<t \leqslant 1$ be such that $r(1+t)<c \operatorname{dist}(x, \partial D)$. Then $y=-\frac{1}{2} t v$ lies in $D$ and

$$
|y-v|=\left(1+\frac{1}{2} t\right) r, \quad|y-u|<\left(1+\frac{1}{2} t\right) r \text { and }|y-w|<\left(1+\frac{1}{2} t\right) r .
$$

This trichotomy arises for either one of $u$ or $w$ equals $-v$, or the triangle inequality is strict. Now,

$$
\left(1+\frac{1}{2} t\right) r<c \operatorname{dist}(x, \partial D)-\frac{1}{2} t r=c \operatorname{dist}(x, \partial D)-c|y-x| \leqslant c \operatorname{dist}(y, \partial D) .
$$

This is a contradiction to the $c$-convexity of $C$ for $u, w \in B^{n}\left(y,\left(1+\frac{1}{2} t\right) r\right)$ and $v$, which lies between $u$ and $w$ on $C$, does not lie in this ball.

4.6 Lemma. Let $u=\left(u_{1}, u_{2}\right)$ and $v=\left(v_{1}, v_{2}\right)$ be points in $R^{2}$ such that

$$
u_{1}=v_{1} \geqslant \frac{1}{2}\left(\left|u_{2}\right|+\left|v_{2}\right|\right) \text {. }
$$

If $w=\left(w_{1}, w_{2}\right)$ is the centre of the circle through $u, v$ and 0 , then $|w| \leqslant \max \{|u|,|v|\}$.

Proof. We may assume that $\left|u_{2}\right| \geqslant\left|v_{2}\right|$. Now $w_{2}=\frac{1}{2}\left(u_{2}+v_{2}\right)$ and so $\left|w_{2}\right| \leqslant\left|u_{2}\right|$. If $w_{1}>u_{1}$ we have, since clearly $w_{1} \geqslant 0$,

$$
\begin{aligned}
|w-u|^{2} & =\left(w_{1}-u_{1}\right)^{2}+\left(w_{2}-u_{2}\right)^{2}=\left(w_{1}-u_{1}\right)^{2}+\left(\frac{1}{2}\left(v_{2}-u_{2}\right)\right)^{2} \\
& \leqslant\left(w_{1}-u_{1}\right)^{2}+\left(\frac{1}{2}\left(\left|v_{2}\right|+\left|u_{2}\right|\right)\right)^{2} \leqslant\left(w_{1}-u_{1}\right)^{2}+u_{1}^{2}<|w|^{2} .
\end{aligned}
$$

This is a contradiction since $u$ and 0 and equidistant from $w$. Thus $u_{1} \geqslant w_{1}$ and so the result follows. 
4.7 Proposition. Let $C \subset D$ be c-convex with respect to balls in D. Let $y \in C$ and let $T_{y}$ be the unique hyperplane associated to y given in Proposition 4.4. Let $N$ be a codimension one hyperplane parallel to $T_{1}$ with

$$
a=\operatorname{dist}\left(T_{y}, N\right) \leqslant \frac{1}{2} c \operatorname{dist}(y, \partial D) .
$$

Then $C \cap N$ contains at most one point of $B^{n}\left(y, \frac{1}{2} c \operatorname{dist}(y, \partial D)\right)$.

Proof. By rotation and translation we can assume that $y=0$ and that $T_{y}=\{x=$ $\left.\left(x_{1}, \ldots, x_{n}\right) \in R^{n}: x_{1}=0\right\}$, so that $N$ is the $\left\{x_{1}=a\right\}$ hyperplane. Let $r$ $=\frac{1}{2} c \operatorname{dist}(y, \partial D)$ and suppose, for contradiction, that there are at least two such points, say $u$ and $v$. By the construction of $T_{v}$, for each $z \in T_{v} \cap S^{n-1}(r)$

$$
\bar{B}^{n}(z, r) \cap C=\{y\} .
$$

We will say that any ball satisfying (4.4) osculates $C$ at $y$. Now consider the two-dimensional plane $P$ through the points $u, v$ and 0 . Considering the intersection of $P$ with the osculating balls and identifying $P$ with $R^{2}$, it is clear that $u$ and $v$ lie in the region

$$
B^{2}(0, r) \backslash\left\{B^{2}((0, r), r) \cup B^{2}((0,-r), r)\right\} .
$$

$T_{y}$ and $N$ parallel imply that if $u=\left(u_{1}, u_{2}\right)$ and $v=\left(v_{1}, v_{2}\right)$, then $u_{1}=v_{1}$. Since $u$ and $v$ lie in the region described above it is easy to see that $u_{1} \geqslant \frac{1}{2}\left(\left|u_{2}\right|+\left|v_{2}\right|\right)$. Thus by Lemma 4.6 if $w$ is the centre of the circle through $u, v$ and 0 , then $|w| \leqslant$ $\max \{|u|,|v|\}<r$. Thus $w \in D$ and $|w|<c \operatorname{dist}(w, \partial D)$. But $B^{n}(w,|w|)$ contains $u, v$ and 0 in its boundary; these are all points of $C$, contradicting Lemma 4.5. The result follows.

Proof of TheOREM 4.3 CONTINUED. By rotation and translation we may assume that

$$
T_{0}=\left\{y=\left(y_{1}, y_{2}, \ldots, y_{n}\right) \in R^{n}: y_{1}=0\right\} .
$$

In these coordinates let $C(t)=\left(C_{1}(t), C_{2}(t), \ldots, C_{n}(t)\right)$ be the arclength parametrisation of $C$. Proposition 4.7 says that in a neighbourhood of $x_{0}=0$

$$
C \cap\left\{y=\left(y_{1}, y_{2}, \ldots, y_{n}\right) \in R^{n}: y_{1}=a\right\}
$$

is at most one point for all $a<r$. This precisely tells us that $C_{1}(t)$ is injective in this neighbourhood, and so a homeomorphism onto its image. Thus we can parametrise $C$ as $C(t)=(t, f(t))$ in a neighbourhood of $x_{0}$, where $f$ is a continuous function on some interval containing 0 . With this parametrisation it suffices to show that $f^{\prime}(0)=0$ to see that $C$ is differentiable at $x_{0}$. Since $C$ is osculated by balls of radius $r$ at $x_{0}$, considering the equations of the osculating spheres easily yields

$$
|f(t)| \leqslant h(t)=r-\left(r^{2}-t^{2}\right)^{1 / 2} .
$$

Thus

$$
0 \leqslant \frac{|f(t)|}{|t|} \leqslant \frac{|h(t)|}{|t|} \rightarrow\left|h^{\prime}(0)\right|=0 \quad \text { as } t \rightarrow 0 .
$$

So $f^{\prime}(0)=0$ and $\left.(d / d t) C\right|_{t=0}=(1,0, \ldots, 0) \neq 0$. Hence $T_{0}$ is the normal hyperplane to $C$ at $x_{0}$ and since $x_{0}$ was arbitrary it follows that $T_{x}$ is the normal hyperplane to $C$ 
at $x$. Next we show that for $x \in C$ and $y \in C \cap B^{n}\left(x, \frac{1}{2} c \operatorname{dist}(x, \partial D)\right)$

$$
\operatorname{dist}\left(x, T_{x} \cap T_{y}\right) \geqslant \frac{1}{4} c \operatorname{dist}(x, \partial D) .
$$

To see this we may assume that $T_{x} \cap T_{y} \neq \varnothing$ and that $z$ is a point of $T_{x} \cap T_{y}$ realizing this distance. If (4.5) is false, then $|x-z|<\frac{1}{4} c \operatorname{dist}(x, \partial D)$ and so $\operatorname{dist}(z, \partial D)>\frac{3}{4} d(x, \partial D)$. Thus $|x-z|<c \operatorname{dist}(z, \partial D)$ and

$$
|y-z| \leqslant|x-z|+|x-y|<\frac{3}{4} c \operatorname{dist}(x, \partial D) \leqslant c \operatorname{dist}(z, \partial D) .
$$

Let $r^{\prime}=\max \{|x-z|,|y-z|\}$. Then either $B^{n}\left(z, r^{\prime}\right)$ osculates one of $x$ or $y$ and contains the other, or osculates both of $x$ and $y$. Both of these conclusions are easily seen to contradict the $c$-convexity of the curve $C$ in much the same way as in the proof of Lemma 4.6. Next let $\phi$ be the angle between the normal hyperplanes $T_{x}$ and $T_{y}$. To establish (4.2) we may assume that $\phi \neq 0$. Let $z$ be as above. Since $y$ lies within the ball $B^{n}\left(x, \frac{1}{2} c \operatorname{dist}(x, \partial D)\right)$ and outside the balls osculating $C$ at $x$, we see immediately that $\angle(x, z, y) \in\left[\phi, \frac{1}{2} \pi\right]$ and that $|y-z|>|x-z|$. Considering the triangle $\langle x, y, z\rangle$ we see, from the above and from (4.5), that

$$
\frac{|x-y|}{\sin \angle(x, z, y)}=\frac{|y-z|}{\sin \angle(y, x, z)} \geqslant|y-z| \geqslant \frac{1}{4} c \operatorname{dist}(x, \partial D) .
$$

Thus

$$
\sin \phi \leqslant \sin \angle(x, z, y) \leqslant 4|x-y| \operatorname{dist}(x, \partial D)^{-1} .
$$

The proof is now complete.

4.8 COROLlary. The geodesics of the quasihyperbolic metric are $C^{1,1}$ and satisfy (4.2) with $c=1$.

4.9 Remark. The proof of Theorem 4.3 is entirely a local problem. It is clear that if $F$ is a compact set on which an $\operatorname{arc} C$ is $c(F)$-convex, then we obtain (4.2) for some $c$ depending only on $F$. In particular we obtain the following result.

4.10 COROLlary. Let $C$ be a rectifiable arc which is locally convex with respect to balls. Then $C$ has Lipschitz continuous derivatives. In particular, if $q$ is a locally Lipschitz density defining a complete metric $d_{q}$, then each $d_{q}$-geodesic has Lipschitz continuous derivatives.

4.11 Example. There is a domain $D \subset R^{n}$ such that $\partial D$ is $C^{\infty}$ and there are infinitely many quasihyperbolic geodesics which are not $C^{2}$. Furthermore there are points in $D$ for which geodesics are not locally unique.

Proof. Let $D=\left\{x=\left(x_{1}, x_{2}, \ldots, x_{n}\right) \in R^{n}:-1 \leqslant x_{2} \leqslant 1\right\}$. We outline the case $n=2$. If $z=(x, y) \in D$, then if $y>0$ one can see that the quasihyperbolic density and the hyperbolic density of the half-space $\{y<1\}$ agree. Thus locally the geodesics must be the same, namely subarcs of circles orthogonal to the boundary. A similar situation occurs when $y<0$. It is clear that the line $\{y=0\}$ is a quasihyperbolic geodesic. We consider the geodesic between the points $(2,1)$ and $(-2,-1)$. Since subarcs of geodesics are geodesics, from the above remarks it must be that $C \cap\{(x, y) \in D: y>0\}$ is a subarc of a circle orthogonal to the line $\{y=1\}$. 
Similarly $C \cap\{(x, y) \in D: y<0\}$ is a subarc of a circle orthogonal to the line $\{y=-1\}$. Since the quasihyperbolic geodesic is $C^{1}$ it is not difficult to see that

$$
\begin{aligned}
C= & \left\{(x, y):(x-1)^{2}+(y-1)^{2}=1 \text { and } 1 \leqslant x \leqslant 2\right\} \\
& \cup\left\{(x, y):(x+1)^{2}+(y+1)^{2}=1 \text { and }-2 \leqslant x \leqslant-1\right\} \\
& \cup\{(x, y): y=0 \text { and }-1 \leqslant x \leqslant 1\} .
\end{aligned}
$$

It is clear that $C$ is not $C^{2}$ at the points $(1,0)$ and $(-1,0)$. It is also clear that there are infinitely many distinct geodesics through $(0,0)$ in the direction $(1,0)$. Notice that on the $\{y=0\}$ line, the density is not differentiable, whilst elsewhere it is smooth. The bifurcation of geodesics is a typical example of the behaviour of geodesics at points where the density is not differentiable.

4.12 Remarks on CURVATURE. Since the quasihyperbolic geodesics need not be $C^{2}$ we need a geometric characterisation of the euclidean curvature of $C^{1,1}$ curves. This can be obtained in terms of the radius of osculating balls, or tangent balls. Let

$$
r_{0}=\sup \left\{r: B^{n}(y, r) \text { osculates } C \text { at } x \text { for all } y \in T_{x} \cap S^{n-1}(x, r)\right\},
$$

where $T_{x}$ is the normal hyperplane to $C$ at $x$. When the curve is $C^{2}$ the sphere of curvature attains $r_{0}$ and so the euclidean curvature of $C$ at $x$ is $K(C, x)=1 / r_{0}$. We take this to be a definition when the curve is at least $C^{1}$. If $C$ is a $c$-convex curve we obtain a lower bound on $r_{0}$, namely $r_{0} \geqslant \frac{1}{2} c \operatorname{dist}(x, \partial D)$. So that

$$
K(C, x) \leqslant \frac{2}{c} \operatorname{dist}(x, \partial D)^{-1} .
$$

Now the right-hand side of (4.8) is $2 / c$ times the quasihyperbolic density at $x$ and so we obtain the following bound on the total euclidean curvature of a $c$-convex curve:

$$
K_{\mathrm{tot}}(C)=\int_{C} K(C, x) d s \leqslant \frac{2}{c} \int_{C} \operatorname{dist}(x, D)^{-1} d s=\frac{2}{c} L_{q}(C),
$$

where $L_{q}(C)$ denotes the quasihyperbolic length of $C$. However for quasihyperbolic geodesics we can improve this by using Proposition 2.4 which says that the 'cone' at $x$ of radius $r<\operatorname{dist}(x, \partial D)$ osculates $C$ at $x$. This is easily seen to give the bound $r_{0} \geqslant \operatorname{dist}(x, \partial D)$, since this is a local problem. Thus, let $C$ be a quasihyperbolic geodesic between the points $x$ and $y$. We then have

$$
\begin{gathered}
K(C, x) \leqslant \operatorname{dist}(x, \partial D)^{-1}, \\
K_{\text {tot }}(C) \leqslant L_{q}(C)=k_{D}(x, y) .
\end{gathered}
$$

To see that (4.10) is sharp one need only consider the hyperbolic metric in the upper half-space, this is the same as the quasihyperbolic metric. Then for any point there is a geodesic through that point with euclidean curvature equal to $\operatorname{dist}(x, \partial D)^{-1}$. Thus (4.11) is infinitesimally sharp. 
5. The decomposition of uniform domains. We state the main result and delay the proof until a few necessary preliminaries have been developed.

5.1 THEOREM. Let $D$ be a uniform domain. Then there is a constant $L$, depending only on the constants of uniformity for $D$, such that for each pair of points $x_{1}, x_{2}$ in $D$ there is an L-bi-Lipschitz embedding $f: \bar{B}^{n}\left(0,\left|x_{1}-x_{2}\right|\right) \hookrightarrow D$ with

$$
\left\{x_{1}, x_{2}\right\} \subset f\left(\bar{B}^{n}\left(0,\left|x_{1}-x_{2}\right|\right)\right) \text {. }
$$

For $v \in S^{n-1}, v \neq e_{1}$ we define $F_{v}: S^{n-1} \rightarrow S^{n-1}$ to be the unique rotation of $S^{n-1}$ such that

(1) $F_{v}\left(e_{1}\right)=v$.

(2) $F_{v}$ leaves everything orthogonal to $e_{1}$ and $v$ fixed.

I wish to thank J. Väisälä for pointing out to me the explicit formula for $F_{v}$ in $[9$, p. 77] and thus simplifying the proof of the following lemma.

5.2 LemmA. Let $u, v \in S^{n-1}$ and suppose that $\left|e_{1}-v\right| \leqslant\left|e_{1}-u\right| \leqslant 2^{1 / 2}$. Then

$$
\sup \left|F_{v}(x)-F_{u}(x)\right| \leqslant 3^{1 / 2}|v-u|,
$$

where the supremum is taken over all $x \in S^{n-1}$.

Proof. The result is trivial on $S^{1}$ and so we consider $S^{n}$ for $n \geqslant 2$. Then

$$
F_{v}(x)=x-\frac{\left(e_{1}+v\right) \cdot x}{1+e_{1} \cdot v}\left(e_{1}+v\right)+2\left(e_{1} \cdot x\right) v .
$$

If $u=e_{1}$, then $v=e_{1}$ and the result follows. Let $w \in S^{n}$ be perpendicular to both $e_{1}$ and $u$. Then

$$
F_{u}(w)=w \quad \text { and } \quad F_{v}(w)=w-\frac{v \cdot w}{1+e_{1} \cdot v}\left(e_{1}+v\right)
$$

so that

$$
\left|F_{u}(w)-F_{v}(w)\right|=\left|v \cdot w \frac{\left(e_{1}+v\right)}{1+e_{1} \cdot v}\right|=\left|(v-u) \cdot w \frac{\left(e_{1}+v\right)}{1+e_{1} \cdot v}\right| \leqslant 2^{1 / 2}|v-u|,
$$

since $\left|e_{1}+v\right|^{2}=2\left(1+e_{1} \cdot v\right)$. Next suppose that $x$ lies on the great circle through $e_{1}$ and $w$. Then $x=c_{1} e_{1}+c_{2} w$, where $c_{1}^{2}+c_{2}^{2}=1$, and thus

$$
\begin{aligned}
\left|F_{u}(x)-F_{v}(x)\right| & \leqslant\left|c_{1}\right|\left|F_{u}\left(e_{1}\right)-F_{v}\left(e_{1}\right)\right|+\left|c_{2}\right|\left|F_{u}(w)-F_{v}(w)\right| \\
& \leqslant\left(\left|c_{1}\right|+2^{1 / 2}\left|c_{2}\right|\right)|v-u| \leqslant 3^{1 / 2}|v-u| .
\end{aligned}
$$

Now consider the rotation $F_{v}^{-1} F_{u}$. This leaves some great circle invariant and $\left|F_{v}^{-1} F_{u}(x)-x\right|$ is constant and maximal on this circle. But since any two great circles must intersect and since we have (5.2) on the great circle through $e_{1}$ and $w$, the lemma follows.

5.3 REMARKS. Lemma 5.2 tells us that we can take Lipschitz sections of the bundle $S O(n) \rightarrow S O(n) / S O(n-1)$. In particular, suppose that $M^{m}$ is a submanifold of $R^{n}$ and that $U \subset M$ is an open subset. Then for any locally Lipschitz $f: U \rightarrow S^{n-1}$ with $f(U) \neq S^{n-1}$ we can lift $f$ to $\bar{f}: U \rightarrow S O(n)$ as a locally Lipschitz map, which can be 
thought of as a Lipschitz frame on $U$. If $M$ is a $C^{1.1}$ manifold, then using a Lipschitz varying orthonormal $m$-frame and composing with similar rotations we can find a locally Lipschitz $n$-frame along $U$. This observation proves a Lipschitz tubular neighbourhood theorem for immersed $C^{1,1}$ submanifolds of $R^{n}$. Let $S_{+}^{n-1}$ denote a hemisphere of $S^{n-1}$. We summarise the above in the following proposition.

5.4 Proposition. Suppose that $U$ is a coordinate patch of a $C^{1.1}$ manifold and that $f: U \rightarrow S_{+}^{n-1}$ is a locally L-Lipschitz map. Then there is a locally $3^{1 / 2}$ L-Lipschitz map $\bar{f}: U \rightarrow S O(n)$ such that the following diagram commutes:

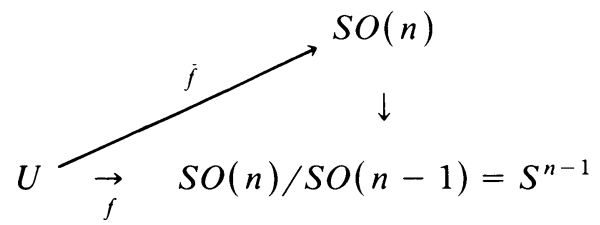

Further, inductively choosing $f$ to be the orthonormal coordinate derivatives we can choose the n-frame to be along $U$.

We now extend each rotation $F_{v}$, radially to be a rotation of $R^{n}$. It is then clear

$$
\left|F_{v}(x)-F_{u}(x)\right| \leqslant 3^{1 / 2}|x||u-v|,
$$

and that each $F_{v}$ is then a linear isometry of $R^{n}$.

Proof of Theorem 5.1. The above remarks are true for $C^{1,1}$ arcs and since quasihyperbolic geodesics are $C^{1,1}$ we construct a nice section of the frame bundle along a quasihyperbolic geodesic to give a Lipschitz tubular neighbourhood and thus the map $f$. Convexity with respect to balls will give the local results while the uniform conditions, which hold along a quasihyperbolic geodesic, will give the global results.

Let $x_{1}$ and $x_{2}$ be points of $D$ and let $C$ be a quasihyperbolic geodesic between them, parametrised by arclength. Thus $C:[0, S] \rightarrow D, C(0)=x_{1}, C(S)=x_{2}$ and $S=l(C)$. Let $a$ and $b$ be the constants of uniformity for $D$ and set $M=100 a b$. Let $H=[0, S] \times \bar{B}^{n-1}\left(M^{-1}\left|x_{1}-x_{2}\right|\right)$. We then define our prototype doublecone domain, on which we base our construction, as

$$
\begin{aligned}
G= & \left\{(t, t x / S):(t, x) \in H \text { and } t \leqslant \frac{1}{2} S\right\} \\
& \cup\left\{(t,(S-t) x / S):(t, x) \in H \text { and } t \geqslant \frac{1}{2} S\right\},
\end{aligned}
$$

and for $0 \leqslant t \leqslant S$ let

$$
G_{t}=G \cap\left\{y=\left(y_{1}, \ldots, y_{n}\right) \in R^{n}: y_{1}=t\right\} .
$$

Then by (3.1)

(5.6) $\operatorname{diam}\left(G_{t}\right)=\frac{4}{M S}\left|x_{1}-x_{2}\right| \min \{t, S-t\} \leqslant \frac{4 b}{M S}\left|x_{1}-x_{2}\right| \operatorname{dist}(C(t), \partial D)$.

It is clear that there is an $L_{1}$-bi-Lipschitz homeomorphism $h: \bar{B}^{n}\left(\left|x_{1}-x_{2}\right|\right) \rightarrow G$, where $L_{1}$ depends only on $a$ and $b$, for by (3.1) $S \leqslant a\left|x_{1}-x_{2}\right|$. Next let $T_{t}=\{y=$ $\left.\left(y_{1}, \ldots, y_{n}\right): y_{1}=t\right\}$, and let $N_{t}$ denote the normal hyperplane to $C$ at $C(t)$. Since $C(t)$ is a $C^{1,1}$ arc parametrised by arclength, $\left|C^{\prime}(t)\right|=1$ and $C^{\prime}:[0, S] \rightarrow S^{n-1}$ is 
locally Lipschitz and hence not spacefilling. We may assume by a rotation of $R^{n}$ that $C^{\prime}\left(\frac{1}{2} S\right)=e_{1}$. We now define a locally Lipschitz collection of rotations of $R^{n}$ that will map the hyperplane orthogonal to $e_{1}$ parallel to $N_{t}$.

Let $t_{j} \in[0, S], j=-m,-m-1, \ldots,-1,0,1, \ldots, n-1, n$, be a finite collection of points such that

(1) $t_{-m}=0, \quad t_{0}=\frac{1}{2} S, \quad t_{n}=S$,

(2) $\left|C^{\prime}\left(t_{j}\right)-C^{\prime}\left(t_{j+1}\right)\right|=2^{1 / 2}$ for $-m<j<n-1$,

(3) $\left|C^{\prime}(t)-C^{\prime}\left(t_{j-1}\right)\right| \leqslant 2^{1 / 2}$ for $t_{j} \leqslant t \leqslant t_{j+1}$ and all $j$.

Suppose that $|t-s|<\frac{1}{8} \operatorname{dist}(C(t), \partial D), t \leqslant r \leqslant s$ and $\phi$ is the angle between the normal hyperplanes $N_{t}$ and $N_{r}$. Then, by (4.2)

$$
\begin{aligned}
\sin (\phi) & \leqslant 4|C(t)-C(r)| \operatorname{dist}(C(t), \partial D)^{-1} \\
& \leqslant 4|t-r| \operatorname{dist}(C(t), \partial D)^{-1}<\frac{1}{2} .
\end{aligned}
$$

Thus $\phi \in[0, \pi / 2]$ and so

$$
\left|C^{\prime}(t)-C^{\prime}(r)\right|=2 \sin \left(\frac{1}{2} \phi\right) \leqslant 2^{1 / 2} \sin (\phi)<2^{-1 / 2} .
$$

Thus,

$\left|C^{\prime}\left(r_{1}\right)-C^{\prime}\left(r_{2}\right)\right| \leqslant\left|C^{\prime}(t)-C^{\prime}\left(r_{1}\right)\right|+\left|C^{\prime}(t)-C^{\prime}\left(r_{2}\right)\right|<2^{1 / 2}, \quad t \leqslant r_{1} \leqslant r_{2} \leqslant s$, and so for no two points $r_{1}, r_{2}$ between $t$ and $s$ is it true that $C^{\prime}\left(r_{1}\right)$ is orthogonal to $C^{\prime}\left(r_{2}\right)$. From (5.7) there is a $j$ so that

$$
t_{j}<t \leqslant s \leqslant t_{j+1} \text { or } t_{j}<t \leqslant t_{j+1} \leqslant s<t_{j+2} .
$$

For $t \in[0, S]$ we define a rotation of $R^{n}$ inductively as follows. Set $F_{t_{0}}=$ identity. We suppose that $t>t_{0}$; the construction for $t<t_{0}$ is similar. There is a $j$ so that $t_{j}<t \leqslant t_{j+1}$. Let $P_{t}$ be the rotation in the great circle through $C^{\prime}\left(t_{j}\right)$ and $C^{\prime}(t)$ as in (5.1) but mapping $C^{\prime}\left(t_{j}\right)$ to $C^{\prime}(t)$. Then set

$$
F_{t}=P_{t} \cdot F_{t_{j}}: S^{n-1} \rightarrow S^{n-1} .
$$

Essentially we are renormalizing the rotations at the points $C^{\prime}\left(t_{j}\right)$ in case that $C^{\prime}$ does not lie in a hemisphere. If we extend each $F_{t}$ radially to a rotation of $R^{n}$, then it is clear that $F_{t}\left(e_{1}\right)=C^{\prime}(t)$ and that $F_{t}$ maps $T_{0}$ parallel to $N_{t}$. We now show for $t$, $s \in[0, S]$ with $|s-t|<\frac{1}{8} \operatorname{dist}(C(t), \partial D)$ and $x \in S^{n-1}$, that

$$
\left|F_{t}(x)-F_{s}(x)\right| \leqslant(96)^{1 / 2} d^{-1}|t-s|,
$$

where $d=\min \{\operatorname{dist}(C(t), \partial D), \operatorname{dist}(C(s), \partial D)\}$. To see this we may assume by symmetry that $t<s$ and $d=\operatorname{dist}(C(s), \partial D)$. By (5.8) there are two cases to consider. We consider the latter, the other case being similar. Now,

$$
\left|F_{t}(x)-F_{s}(x)\right| \leqslant\left|F_{t}(x)-F_{t_{j+1}}(x)\right|+\left|F_{s}(x)-F_{t_{j+1}}(x)\right| .
$$


Since $F_{t}$ and $F_{t_{j+1}}$ are both rotations through great circles through $C^{\prime}\left(t_{j}\right)$ we obtain from Lemma 5.2 replacing $e_{1}$ by $C^{\prime}\left(t_{j}\right)$,

$$
\begin{aligned}
\left|F_{t}(x)-F_{t_{j+1}}(x)\right| & \leqslant 3^{1 / 2}\left|F_{t}\left(C^{\prime}\left(t_{j}\right)\right)-F_{t_{j+1}}\left(C^{\prime}\left(t_{j}\right)\right)\right| \\
& =3^{1 / 2}\left|C^{\prime}(t)-C^{\prime}\left(t_{j+1}\right)\right|=3^{1 / 2} 2 \sin \left(\frac{1}{2} \phi\right) \\
& \leqslant 3^{1 / 2} 2^{1 / 2} \sin (\phi) \leqslant(96)^{1 / 2}\left|C(t)-C\left(t_{j+1}\right)\right| \operatorname{dist}(C(t), \partial D)^{-1} \\
& \leqslant(96)^{1 / 2}\left|t-t_{j+1}\right| \operatorname{dist}(C(t), \partial D)^{-1} .
\end{aligned}
$$

This follows from (4.2) and the above. A similar estimate holds for $F_{s}$ and $F_{t_{t+1}}$, and, since $\left|t-t_{j+1}\right|+\left|t_{j+1}-s\right|=|t-s|,(5.10)$ easily follows. Thus the $F_{t}$ form a locally $(96)^{1 / 2}$-Lipschitz frame along $C$.

Next let $H_{t}(x)=F_{t}(x-(t, 0, \ldots, 0))+C(t)$ for $t \in[0, S]$, and let $g: G \rightarrow D$ be the map

$$
g(x)=H_{t}(x) \text { for } x \in G_{t} .
$$

Now each $H_{t}$ is an isometry and since $G=\cup_{t} G_{t}, g$ is well defined and by (5.6), $g(G) \subset D$. We have three things to establish:

$$
\begin{aligned}
& \text { (1) } g \text { is injective, } \\
& \text { (2) } g \text { is locally bi-Lipschitz, } \\
& \text { (3) } g \text { is globally bi-Lipschitz. }
\end{aligned}
$$

(1) It is clear that $g$ is injective on each $G_{t}$. By construction $H_{t}\left(G_{t}\right)$ and $H_{s}\left(G_{s}\right)$ lie on the normal hyperplanes to $C$ at $C(t)$ and $C(s)$ respectively. Since $C$ is a quasihyperbolic geodesic, these hyperplanes cannot intersect within $\frac{1}{4} \operatorname{dist}(C(t), \partial D)$ unless $|C(t)-C(s)| \geqslant \frac{1}{2} \operatorname{dist}(C(t), \partial D)$, by (4.5). By (5.6)

$$
\operatorname{diam}\left(H_{t}\left(G_{t}\right)\right)=\operatorname{diam}\left(G_{t}\right) \leqslant(25 a)^{-1} \operatorname{dist}(C(t), \partial D),
$$

and similarly for $\operatorname{diam}\left(H_{s}\left(G_{s}\right)\right)$. Thus $g$ is injective, since $H_{t}\left(G_{t}\right) \cap H_{s}\left(G_{s}\right)=\varnothing$.

(2) Let $r(t)=(8 b)^{-1} \min \{t, S-t\} \leqslant 8^{-1} \operatorname{dist}(C(t), \partial D)$.

We show that if $x \in G_{t}$ and $y \in G_{s}$ with $|t-s| \leqslant r(t)$, then

$$
(3 a)^{-1}|x-y| \leqslant|g(x)-g(y)| \leqslant 3|x-y| \text {. }
$$

By symmetry we may assume that $\operatorname{dist}(C(s), \partial D) \leqslant \operatorname{dist}(C(t), \partial D)$. Let

$$
x_{0}=\left(0, x_{2}, \ldots, x_{n}\right), \quad y_{0}=\left(0, y_{2}, \ldots, y_{n}\right)
$$

so that $x_{0}, y_{0} \in T_{0}$. Then,

$$
\begin{aligned}
|g(x)-g(y)| & =\left|H_{t}(x)-H_{s}(y)\right| \\
& =\left|F_{t}(x-(t, 0, \ldots, 0))-F_{s}(y-(s, 0, \ldots, 0))+C(t)-C(s)\right| \\
& =\left|F_{t}\left(x_{0}\right)-F_{s}\left(y_{0}\right)+z\right|,
\end{aligned}
$$

where $z=C(t)-C(s)$. By (5.10)

$$
\begin{aligned}
\left|F_{t}\left(y_{0}\right)-F_{s}\left(y_{0}\right)\right| & \leqslant(96)^{1 / 2}\left|y_{0}\right||t-s| \operatorname{dist}(C(s), \partial D)^{-1} \\
& \leqslant \frac{1}{5 a}|t-s|
\end{aligned}
$$


for $\left|y_{0}\right| \leqslant \frac{1}{2} \operatorname{diam}\left(G_{s}\right) \leqslant(50 \mathrm{a})^{-1} \operatorname{dist}(C(s), \partial D)$. Thus by the triangle inequality

$$
\begin{aligned}
|g(x)-g(y)| & \leqslant\left|F_{t}\left(x_{0}\right)-F_{t}\left(y_{0}\right)\right|+\left|F_{t}\left(y_{0}\right)-F_{s}\left(y_{0}\right)\right|+|z| \\
& \leqslant\left|x_{0}-y_{0}\right|+(5 a)^{-1}|t-s|+|t-s| \leqslant 3|x-y| .
\end{aligned}
$$

Similarly,

$$
|g(x)-g(y)| \geqslant\left|z+F_{t}\left(x_{0}\right)-F_{t}\left(y_{0}\right)\right|-(5 a)^{-1}|x-y| .
$$

Now $F_{t}\left(x_{0}-y_{0}\right)$ lies in the hyperplane through the origin parallel to $N_{t}$ and so $C(t)+F_{t}\left(x_{0}-y_{0}\right)$ lies in $N_{t}$. Also $C(s)$ lies on $N_{s}$ and

$$
|C(t)-C(s)| \leqslant|t-s| \leqslant r(t) \leqslant \frac{1}{8} \operatorname{dist}(C(t), \partial D),
$$

so $C(s)$ lies between the osculating balls to $C$ at $C(t)$ of radius $\frac{1}{2} \operatorname{dist}(C(t), \partial D)$. One can then see that the angle between the line through $C(t)$ and $C(s)$, and $N_{t}$ is between $\pi / 3$ and $5 \pi / 6$. Considering the triangle $\left\langle C(t)+F_{t}\left(x_{0}-y_{0}\right), C(t), C(s)\right\rangle$ by the law of cosines we see,

$$
\begin{aligned}
\left|z+F_{t}\left(x_{0}-y_{0}\right)\right|^{2} \geqslant & \left|x_{0}-y_{0}\right|^{2}+|C(s)-C(t)|^{2} \\
& -2\left|x_{0}-y_{0}\right||C(s)-C(t)| \cos (\pi / 3) \\
\geqslant & \frac{1}{2}(|x-y| / a)^{2} .
\end{aligned}
$$

For $|t-s| \leqslant a|C(t)-C(s)|$ and $\left|x_{0}-y_{0}\right|^{2}+|t-s|^{2}=|x-y|^{2}$. This together with (5.16) yields

$$
|g(x)-g(y)| \geqslant(3 a)^{-1}|x-y| .
$$

This establishes (5.14) and so $g$ is a locally $3 a$-bi-Lipschitz embedding.

(3) Since $G$ is convex, if $x, y \in G$ then the line segment between $x$ and $y$ lies in $G$ and the image of this line segment has length no more than $3|x-y|$ for locally $g$ is 3-Lipschitz, and $g([x, y])$ joins $g(x)$ to $g(y)$. Thus

$$
|g(x)-g(y)| \leqslant 3|x-y| \text {. }
$$

Next suppose that $x \in G_{t}$ and $y \in G_{s}$ with $|s-t| \geqslant \max \{r(t), r(s)\}$, otherwise (5.14) yields the result. Since $g$ is an isometry on each $G_{t}$ we have

$$
\begin{aligned}
|g(x)-g(y)| & \geqslant|g((t, 0, \ldots, 0))-g((x, 0, \ldots, 0))|-\frac{1}{2} \operatorname{diam}\left(G_{t}\right)-\frac{1}{2} \operatorname{diam}\left(G_{s}\right) \\
& \geqslant|C(t)-C(s)|-(3 a)^{-1}|t-s| \geqslant(2 a)^{-1}|t-s| .
\end{aligned}
$$

Then

$$
\operatorname{diam}\left(G_{t}\right)=\frac{4}{M S} \min \{t, S-t\}\left|x_{1}-x_{2}\right| \leqslant(3 a)^{-1} r(t) \leqslant(3 a)^{-1}|t-s|,
$$

and similarly for $\operatorname{diam}\left(G_{s}\right)$. Next,

$$
|x-y| \leqslant|s-t|+\frac{1}{2} \operatorname{diam}\left(G_{t}\right)+\frac{1}{2} \operatorname{diam}\left(G_{s}\right) \leqslant \frac{3}{2}|s-t| .
$$

This together with the above yields

$$
|g(x)-g(y)| \geqslant(3 a)^{-1}|x-y| .
$$

Thus we have

$$
(3 a)^{-1}|x-y| \leqslant|g(x)-g(y)| \leqslant 3|x-y| .
$$


It is now clear that $f=g \cdot h: \bar{B}^{n}\left(\left|x_{1}-x_{2}\right|\right) \rightarrow R^{n}$ is the desired bi-Lipschitz embedding to establish Theorem 5.1.

5.4 RemarKs. (1) It is not difficult to explicitly construct the map $h$ and estimate the associated Lipschitz constant to be less than $200 \mathrm{ab}$. Thus (5.17) yields

$$
\left(600 a^{2} b\right)^{-1}|x-y| \leqslant|f(x)-f(y)| \leqslant 600 a b|x-y| .
$$

The right estimate appears to have the correct order whilst the left does not.

(2) We recover Theorem 3.4 when $n=2$, because each bi-Lipschitz map of a disk extends to a quasiconformal homeomorphism of $\bar{R}^{2}$, and the dilatation of the extension depends only on the Lipschitz constants [1].

(3) Since bi-Lipschitz balls are uniform domains it is clear that Theorem 5.1 holds in a domain $D$ if and only if $D$ is uniform.

(4) Since the construction was based on a quasihyperbolic geodesic which is a tame arc, it is clear that $f\left(\bar{B}^{n}\left(\left|x_{1}-x_{2}\right|\right)\right)$ is tame and so has a topological extension. Example 3.8 shows that there is a topological obstruction to this extension being quasiconformal with dilatation depending only on the Lipschitz constants, when $n=3$. Theorem 3.7 is true in all dimensions greater than three. Indeed we can suspend the map of 3.7 on the points $(0,0,0, \pm 1)$ in $R^{4}$. Since the map of 3.7 was not one locally coconnected neither will be the suspension [2, 4A]. It is easy to see that the suspension will be bi-Lipschitz. We can now induct on this construction to provide higher dimensional examples. However such examples do not provide counterexamples to higher dimensional analogues to Theorem 3.4, since in our construction each map $g_{j}$ has an extension and as in 3.8 the limit of such maps may be forced to be wild at two points, this is the basis of 3.8. However when $n \geqslant 4$ wildness at a discrete set is not an obstruction to an extension [2, Corollary 3A.5]. Thus there is no topological obstruction to an extension which is quasiconformal with dilatation depending only on the Lipschitz constant. One is led to conjecture that higher dimensional analogues to 3.4 might be true.

(5) [ADDED IN PROOF] Recently I have found examples, for all $n>2$, of topologically flat $(n-1)$-spheres in $R^{n}$ which are locally $K$-quasiconformally flat except at one point (for all $K>1$ ) and not quasiconformally flat for any finite dilatation. Thus bad behaviour (in a quasiconformal sense) at a discrete set is not necessarily a removable condition in the quasiconformal category, see [12]. The main feature of these examples is that the complementary domains of these $(n-1)$-spheres are uniform. It then seems that the higher dimensional analogues of Theorem 3.4 are also false for $n>3$.

\section{REFERENCES}

1. L. V. Ahlfors, Quasiconformal reflections, Acta Math. 109 (1963).

2. R. Daverman, Embeddings of $(n-1)$-spheres in euclidean n-space, Bull. Amer. Math. Soc. 84 (1978).

3. F. W. Gehring, Injectivity of local quasi-isometries, Comment. Math. Helv. 57 (1982).

4. F. W. Gehring and B. Osgood, Uniform domains and the quasihyperbolic metric, J. Analyse Math. 36 (1979).

5. F. W. Gehring and B. Palka, Quasiconformally homogeneous domain, J. Analyse Math. 30 (1976).

6. F. W. Gehring and J. Väisälä, The coefficients of quasiconformality of domains in space. Acta Math. $114(1965)$ 
7. P. W. Jones, Extension theorems for BMO, Indiana Univ. Math. J. 29 (1980).

8. O. Martio and J. Sarvas, Injectivity theorems in plane and space, Ann. Acad. Sci. Fenn. Ser. AI 4 $(1978 / 1979)$.

9. J. W. Milnor and J. D. Stasheff, Characteristic classes, Ann. of Math. Studies, no. 76, Princeton Univ. Press, Princeton, N. J., 1974.

10. T. B. Rushing, Topological embeddings, Academic Press, New York, 1973.

11. J. Väisälä, Lectures on n-dimensional quasiconformal mappings, Lecture Notes Math., vol. 229, Springer-Verlag, Berlin and New York, 1971.

12. G. J. Martin, Quasiconformal groups which are not the quasiconformal conjugates of Möbius groups (to appear).

Department of Mathematics, University of Michigan, Ann Arbor, Michigan 48109

Current address: Mathematical Sciences Research Institute, 1000 Centennial Drive, Berkeley, California 94720 